PROCEEDINGS OF THE

AMERICAN MATHEMATICAL SOCIETY

Volume 140, Number 1, January 2012, Pages 199-206

S 0002-9939(2011)10988-2

Article electronically published on May 11, 2011

\title{
THE C*-ALGEBRA OF A PARTIAL ISOMETRY
}

\author{
BERNDT BRENKEN AND ZHUANG NIU \\ (Communicated by Marius Junge)
}

\begin{abstract}
The universal $\mathrm{C}^{*}$-algebra generated by a partial isometry is a nonunital residually finite dimensional $\mathrm{C}^{*}$-algebra which is not exact. Many unitarily inequivalent partial isometries generating any given finite dimensional full matrix algebra are constructed. The $K$-groups of this algebra are computed, and it is shown that all projections in the algebra are equivalent.
\end{abstract}

\section{INTRODUCTION}

Partial isometries play a basic and ubiquitous role in several aspects of Operator Algebras, as the type decomposition of von Neumann algebras or the $K$-theory of operator algebras illustrates. Universal $\mathrm{C}^{*}$-algebras generated by families of partial isometries subject to certain relations involving source and range projections provide many fundamental examples of $\mathrm{C}^{*}$-algebras and have been the subject of much recent investigation $([\mathrm{B}],[\mathrm{R}])$. On the other hand, the universal $\mathrm{C}^{*}$-algebra generated by a single partial isometry may generally be viewed as intractable ([HM], $\mathrm{P})$. We further document this aspect and aim to show that it is nevertheless an interesting algebra. We show that this algebra is intimately related, in particular Morita equivalent, to the universal unital $\mathrm{C}^{*}$-algebra generated by a contraction. It follows that it is a residually finite dimensional $\mathrm{C}^{*}$-algebra which is not exact. There are other known examples of this: the $\mathrm{C}^{*}$-algebra of the free group on a set with more than one element provides (unital) examples. There are many unitarily inequivalent partial isometries singly generating any finite dimensional full matrix algebra. The $K$-groups of this algebra coincide with the $K$-groups of the Toeplitz algebra; although the projections in the algebra are tightly constrained, they are all equivalent. In particular the algebra is nonunital. The algebra also contains an ideal which is in some aspects analogous to the ideal of compacts in the Toeplitz algebra.

We are grateful to C. Phillips for pointing out the reference $\mathrm{OZ}$.

\section{Notation}

For $\mathcal{H}$ a Hilbert space, $B(\mathcal{H})$ denotes the $\mathrm{C}^{*}$-algebra of bounded operators on

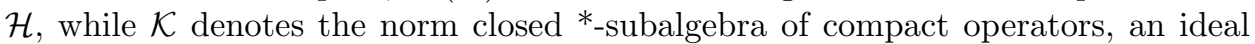
in $B(\mathcal{H})$, where ideal means a closed two-sided ideal. For $A \in B(\mathcal{H})$ the point spectrum of $A$ is $\sigma_{p}(A)$. For $X \subseteq \mathcal{H},[X]$ is the smallest closed subspace of $\mathcal{H}$ containing $X$. If $S$ is a subset of $B(\mathcal{H})$, then $S^{\prime}$, the commutant of $S$, is the

Received by the editors October 1, 2009 and, in revised form, November 3, 2010.

2010 Mathematics Subject Classification. Primary 46L35, 46L80, 47C15.

(C)2011 American Mathematical Society 
collection of operators in $B(\mathcal{H})$ commuting with $S \cup S^{*}$. For a $\mathrm{C}^{*}$-algebra $\mathcal{A}$ and $n \in \mathbb{N}, \mathrm{M}_{n}(\mathcal{A})$ is the $\mathrm{C}^{*}$-algebra of $n$ by $n$ matrices with entries in $\mathcal{A}$, and $\widetilde{\mathcal{A}}$ denotes the unitization of $\mathcal{A}$. If $\mathcal{A}$ is a set, then $I d_{\mathcal{A}}$ denotes the identity map of $\mathcal{A}$ to itself, while if $\mathcal{A}$ is a unital algebra, $1_{\mathcal{A}}$ denotes the unit. The open unit disk in the complex plane $\mathbb{C}$ is denoted by $D$.

\section{The $\mathrm{C}^{*}$-Algebra of a PARTial isometry}

Denote by $\mathcal{P}$ the universal $\mathrm{C}^{*}$-algebra $([\mathrm{B}]$ ) generated by a partial isometry $v$ and denote the initial projection $v^{*} v$ by $p$ and the final projection $v v^{*}$ by $q$. In $[\mathrm{P}$. it is shown that there is a partial isometry generating a type $\mathrm{II}_{1}$ factor, namely the $\mathrm{II}_{1}$ factor associated with the faithful tracial state on an irrational rotation algebra, showing that $\mathcal{P}$ is not type $\mathrm{I}$.

The following result can be viewed as extending an early result of Halmos and McLaughlin $(\underline{\mathrm{HM}}]$ ). Denote by $\mathcal{D}$ the universal unital (with unit $1_{\mathcal{D}}$ ) $\mathrm{C}^{*}$-algebra generated by a single element $a$ of norm less than or equal to 1.

Theorem 2.1. The $C^{*}$-algebra $\mathcal{P}$ is stably isomorphic, and thus Morita equivalent, to $\mathcal{D}$.

Proof. Since $v p=v$ the ideal of $\mathcal{P}$ generated by $p$ is $\mathcal{P}$; hence $\mathcal{P}$ is stably isomorphic to the hereditary unital sub-C ${ }^{*}$-algebra $p \mathcal{P} p$ by Brown's theorem. Therefore it is enough to show that $p \mathcal{P} p$ is isomorphic to $\mathcal{D}$.

Since the $\mathrm{C}^{*}$-algebra $p \mathcal{P} p$ is generated by

$$
\left\{p v^{n} p ; n \in \mathbb{N} \cup\{0\}\right\}
$$

and

$$
p v^{n} p=p v^{n}=(p v)^{n}=(p v p)^{n} \quad \text { for any } n \in \mathbb{N},
$$

we have that $p \mathcal{P} p$ is generated by $\{p, p v p\}$. Therefore there is a unital surjective *-homomorphism $\phi: \mathcal{D} \rightarrow p \mathcal{P} p$ with $\phi(a)=p v p$.

On the other hand, the element

$$
v_{a}=\left[\begin{array}{cc}
a & 0 \\
\sqrt{1_{\mathcal{D}}-a^{*} a} & 0
\end{array}\right]
$$

is a partial isometry in $\mathrm{M}_{2}(\mathcal{D})$, so there is a *homomorphism $\psi^{\prime}: \mathcal{P} \rightarrow \mathrm{M}_{2}(\mathcal{D})$ with $\psi^{\prime}(v)=v_{a}$. Since $v_{a}^{*} v_{a}=\operatorname{diag}\left\{1_{\mathcal{D}}, 0\right\}$, the cut-down of $\psi^{\prime}$ by $p=v^{*} v$ defines a unital *-homomorphism $\psi: p \mathcal{P} p \rightarrow \mathcal{D}$ with $\psi(p v p)=a$. We have that

$$
\psi \circ \phi=I d_{\mathcal{D}}
$$

In particular, the map $\phi$ is injective, and hence $\phi$ is a *-isomorphism.

Note that since $\psi$ is an isomorphism of the full hereditary subalgebra $p \mathcal{P} p$ of $\mathcal{P}$, then $\psi^{\prime}: \mathcal{P} \rightarrow \mathrm{M}_{2}(\mathcal{D})$ is also an injection.

Many properties of the two universal algebras $\mathcal{P}$ and $\mathcal{D}$ are therefore shared. Since $\mathcal{P}$ is described via an algebraic condition while $\mathcal{D}$ involves an analytic condition it is hoped that a better understanding of both algebras can result from the flexibility provided between these two conditions. Various properties of $\mathcal{D}$ may be known or clear to some but are included here for completeness.

Corollary 1. The $C^{*}$-algebra $\mathcal{P}$ is not exact. 
Proof. By Theorem 2.1 it is enough to notice that the $\mathrm{C}^{*}$-algebra $\mathcal{D}$ is not exact. Since exactness passes to quotients it suffices to know that there is a singly generated $\mathrm{C}^{*}$-algebra which is not exact. Consider a faithful representation of the nonexact full group $\mathrm{C}^{*}$-algebra $C^{*}\left(\mathbb{F}_{2}\right) \subset B(\mathcal{H})$. If $u$ and $v$ denote the canonical generators, set $h_{u}$ and $h_{v}$ to be the logarithms of $u$ and $v$ respectively in $B(\mathcal{H})$. Since exactness also passes to sub- $\mathrm{C}^{*}$-algebras and the singly generated $\mathrm{C}^{*}$-algebra generated by $w:=h_{u}+\sqrt{-1} h_{v}$ contains $C^{*}\left(\mathbb{F}_{2}\right)$, it is not exact.

Since subalgebras of a residually finite dimensional algebra are also such and matrix algebras over a residually finite dimensional algebra are again residually finite dimensional, the results of Theorem 2.1 show that $\mathcal{P}$ is residually finite dimensional if and only if $\mathcal{D}$ is. The argument of [Ch] carries over easily to either algebra and is included for the sake of completeness.

Theorem 2.2. The $C^{*}$-algebra $\mathcal{P}$ has a countable family of finite dimensional representations $\left\{\pi_{n}\right\}$ such that $\bigoplus \pi_{n}$ is a faithful representation of $\mathcal{P}$. In particular, the $C^{*}$-algebra $\mathcal{P}$ is residually finite dimensional and has a faithful tracial state.

Proof. Since $\mathcal{P}$ is separable, there is a faithful representation $\rho$ on a separable Hilbert space $\mathcal{H}$. Denote $\rho(v)$ by $V, \rho\left(v v^{*}\right)$ by $Q$. Since $Q$ is a projection, there is a sequence of finite dimensional projections $\left(P_{n}\right)$ such that each $P_{n}$ commutes with $Q$, and $\left(P_{n}\right)$ converges in the strong operator topology to $\mathbf{1} \in B(\mathcal{H})$. Denote by $V_{n}$ and $Q_{n}$ the finite rank operators $P_{n} V P_{n}$ and $P_{n} Q P_{n}$ respectively. Noting that

$$
V_{n} V_{n}^{*}=P_{n} V P_{n} V^{*} P_{n} \leq P_{n} V V^{*} P_{n}=P_{n} Q P_{n}=Q_{n}
$$

and that $Q_{n}$ is a projection, we have that the operator

$$
W_{n}:=\left[\begin{array}{cc}
V_{n} & \left(Q_{n}-V_{n} V_{n}^{*}\right)^{1 / 2} \\
0 & 0
\end{array}\right]
$$

is a partial isometry acting on the finite dimensional subspace $P_{n} \mathcal{H} \oplus P_{n} \mathcal{H}$ of $\mathcal{H} \oplus \mathcal{H}$. By the universality of $\mathcal{P}$, there is a *homomorphism

$$
\pi_{n}: \mathcal{P} \rightarrow B\left(P_{n} \mathcal{H} \oplus P_{n} \mathcal{H}\right)
$$

induced by

$$
v \mapsto W_{n} .
$$

Since $P_{n}$ converges to 1 in the strong operator topology, the partial isometries $W_{n}$ converge to

$$
\left[\begin{array}{ll}
V & 0 \\
0 & 0
\end{array}\right]
$$

in the strong operator topology on $B(\mathcal{H} \oplus \mathcal{H})$. Therefore, for any polynomial $g(x, y)$ without constant term we have

$$
\pi_{n}\left(g\left(v, v^{*}\right)\right)=g\left(W_{n}, W_{n}^{*}\right) \rightarrow\left[\begin{array}{cc}
g\left(V, V^{*}\right) & 0 \\
0 & 0
\end{array}\right]
$$

in the strong operator topology. Since $\left\|\pi_{n}\left(g\left(v, v^{*}\right)\right)\right\| \leq\left\|g\left(v, v^{*}\right)\right\|=\left\|\rho\left(g\left(v, v^{*}\right)\right)\right\|=$ $\left\|g\left(V, V^{*}\right)\right\|$, we have that $\left\|g\left(W_{n}, W_{n}^{*}\right)\right\|$ converges to $\left\|\rho\left(g\left(v, v^{*}\right)\right)\right\|$, and $\left\|\pi_{n}(x)\right\| \rightarrow$ $\|\rho(x)\|=\|x\|$ for $x$ in a dense subalgebra of $\mathcal{P}$. Defining $\pi$ to be the block diagonal representation

$$
\pi=\bigoplus \pi_{n}
$$

of $\mathcal{P}$, we have that $\pi$ is an isometry, so a faithful representation of $\mathcal{P}$. 
For each $n$ the usual faithful tracial state on $B\left(P_{n} \mathcal{H} \oplus P_{n} \mathcal{H}\right)$ restricted to $\pi_{n}(\mathcal{P})$ defines, after possible normalization, a separating family of tracial states $\tau_{n}$ on $\mathcal{P}$; their sum with suitable scalar coefficients yields a faithful tracial state $\tau$ on $\mathcal{P}$.

This shows that there are no nonzero ${ }^{*}$-homomorphisms from $\mathcal{K}$ to $\mathcal{P}$ since otherwise there would be a nonzero ${ }^{*}$-homomorphism of the simple $\mathrm{C}^{*}$-algebra $\mathcal{K}$ to some finite matrix algebra. The existence of a faithful tracial state implies that $\mathcal{P}$ is a finite $\mathrm{C}^{*}$-algebra and that $\mathcal{P}$, as well as any ideal of $\mathcal{P}$, cannot be stable.

Corollary 2. There is a faithful representation $\pi$ of the $C^{*}$-algebra $\mathcal{P}$ on a separable Hilbert space $\mathcal{H}$ with $\pi(v)=U \pi(p)$ for a unitary $U$ in the weak operator closure of $\pi(\mathcal{P})$.

Proof. Let $\pi_{\alpha}$ be a countable separating family of inequivalent finite dimensional irreducible representations of $\mathcal{P}$ on $\mathcal{H}_{\alpha}$. Since the projection $p_{\alpha}=\pi_{\alpha}(p)$ is equivalent to $\pi_{\alpha}(q)$ in the finite dimensional space $\mathcal{H}_{\alpha}$, there is a unitary $U_{\alpha}$ on $\mathcal{H}_{\alpha}$ with $U_{\alpha} p_{\alpha}=\pi_{\alpha}(v)$. Let $\pi=\bigoplus \pi_{\alpha}$ and $U=\bigoplus U_{\alpha}$.

It immediately follows that the universal $\mathrm{C}^{*}$-algebra generated by a unitary and a projection is not exact. We also see, by considering this unitary and the (symmetric) unitary operator associated with a projection, that there is a quotient of the universal $\mathrm{C}^{*}$-algebra of the free group on two generators that contains $\mathcal{P}$.

We point out some elementary properties of representations $\pi: \mathcal{P} \rightarrow B(\mathcal{H})$. Since $\|\pi(p)\|=\|\pi(v)\|^{2}$ the projection $\pi(p)$ is never zero unless $\pi=0$. If $\pi$ is a nondegenerate representation of $\mathcal{P}$ and $\mathcal{R}$ is the von Neumann algebra generated by $\pi(\mathcal{P})$, we show that $\pi(p) \vee \pi(q)$ is the unit $I d_{\mathcal{H}}$ of $\mathcal{R}$. Denoting the projection onto the closed range of $T \in B(\mathcal{H})$ by $R(T)$, then $R\left(T^{*} T\right)=R\left(T^{*}\right)$ ([KR $)$ and $R(T S) \leq R(T)$ for $S \in B(\mathcal{H})$. Thus $\pi(p)=R\left(\pi(v)^{*}\right), \pi(q)=R(\pi(v))$ and $R(W) \leq$ $\pi(p) \vee \pi(q)$ for any word $W$ in $\pi(v)$ and $\pi\left(v^{*}\right)$ and therefore for any element $W$ of $\pi(\mathcal{P})$. Thus $I d_{\mathcal{H}}=[\pi(\mathcal{P}) \mathcal{H}] \leq \pi(p) \vee \pi(q) \leq I d_{\mathcal{H}}$. Since necessarily $\operatorname{dim}(\pi(p))=$ $\operatorname{dim}(\pi(q))$ (as subspaces of $B(\mathcal{H})$ ), we therefore have $\operatorname{dim}(\pi(p)) \geq \operatorname{dim}(\mathcal{H}) / 2$. Note also ([KR] ) that $R(\pi(p)+\pi(q))=\pi(p) \vee \pi(q)$.

Corollary 1 implies that $\mathcal{P}$ is not $n$-subhomogeneous for any $n$, in contrast to the universal $\mathrm{C}^{*}$-algebra generated by two projections which is 2 -subhomogeneous ([B]). In fact when $\mathcal{H}$ is finite dimensional, $B(\mathcal{H})$ is singly generated $([\mathrm{OZ})$, so there are irreducible representations of $\mathcal{D}$, and therefore of $\mathcal{P}$, on any finite dimensional Hilbert space. The representation of $\mathcal{P}$ as the Toeplitz algebra on an infinite dimensional Hilbert space yields an infinite dimensional irreducible representation. If $\pi$ is an irreducible representation on a finite dimensional $\mathcal{H}$ and $\operatorname{dim}(\mathcal{H})>1$, then the partial isometry $\pi(v)$ must be nonunitary, and we have $\operatorname{dim}(\mathcal{H}) / 2 \leq \operatorname{dim} \pi(p)<\operatorname{dim}(\mathcal{H})$. For $\mathcal{H}$ of finite dimension $k$ the Jordan block of order $k$ is a partial isometry which clearly defines an irreducible representation of $\mathcal{P}$.

We briefly describe some other approaches to forming irreducible representations of $\mathcal{P}$ on a finite dimensional Hilbert space $\mathcal{H}$, which also serve as further examples of partial isometries which singly generate $B(\mathcal{H})$.

If $\mathcal{H}=\mathcal{H}_{0} \oplus \mathcal{H}_{0}$ has even (finite) dimension, choose an $a \in B\left(\mathcal{H}_{0}\right)$ generating $B\left(\mathcal{H}_{0}\right)$ as a $\mathrm{C}^{*}$-algebra ([OZ]). Dividing by a suitable scalar ensures that $\|a\|<1$ and that $I_{B\left(\mathcal{H}_{0}\right)}-a^{*} a$ is invertible. Consider the partial isometry $v_{a} \in \mathrm{M}_{2}\left(B\left(\mathcal{H}_{0}\right)\right)$ in Theorem 2.1 above, with initial projection $e=\operatorname{diag}\left\{I_{B\left(\mathcal{H}_{0}\right)}, 0\right\}$; it is enough to show 
that $C^{*}\left(v_{a}\right)^{\prime}=\mathbb{C} I_{B(\mathcal{H})}$. Since $\mathcal{H}$ is finite dimensional, $C^{*}\left(v_{a}\right)=C^{*}\left(v_{a}\right)^{\prime \prime}$, which contains $e$ and $I_{B(\mathcal{H})}-e$, so any element $b$ of $C^{*}\left(v_{a}\right)^{\prime}$ is block diagonal $\operatorname{diag}\{r, s\}$ for some $r, s \in B\left(\mathcal{H}_{0}\right)$. Since $b$ commutes with $v_{a}$ and $v_{a}^{*}$, we have $r \in C^{*}(a)^{\prime}$ along with $\left(\sqrt{I_{B\left(\mathcal{H}_{0}\right)}-a^{*} a}\right) r=s \sqrt{I_{B\left(\mathcal{H}_{0}\right)}-a^{*} a}$. Thus $(r-s) \sqrt{I_{B\left(\mathcal{H}_{0}\right)}-a^{*} a}=0$, and since $\sqrt{I_{B\left(\mathcal{H}_{0}\right)}-a^{*} a}$ is invertible, $r=s$. However, $C^{*}(a)^{\prime}=\mathbb{C} I_{B\left(\mathcal{H}_{0}\right)}$, so $r$ is a scalar and $b \in \mathbb{C} I_{B(\mathcal{H})}$.

Proposition 1. If $\pi$ is an irreducible representation of $\mathcal{P}$ on a finite dimensional Hilbert space $\mathcal{H}$ of dimension greater than 1 , then there is a family of inequivalent irreducible representations of $\mathcal{P}$ on $\mathcal{H} \oplus \mathbb{C}$ which dilate $\pi$.

Proof. Let $V=\pi(v)$. Since an irreducible representation of a unitary must be one dimensional we have that $V V^{*} \neq I_{B(\mathcal{H})}$, so there is a nonzero $h \in B(\mathcal{H})$, $h \perp V V_{B(\mathcal{H})}^{*}$. For each scalar $\lambda \in D, \lambda \notin \sigma_{p}\left(V^{*}\right)$, normalize $h$ so that $\|h\|^{2}+|\lambda|^{2}=$ 1. Then $W_{\lambda}=\left[\begin{array}{ll}V & h \\ 0 & \lambda\end{array}\right]$ is a partial isometry on $\left.\mathcal{H}_{1}=\mathcal{H} \oplus \mathbb{C}(\underline{\mathrm{HM}}]\right)$. If $B=$ $\left[\begin{array}{ll}A & c \\ r & \mu\end{array}\right] \in B\left(\mathcal{H}_{1}\right)$ commutes with $W_{\lambda}$, an inspection of the lower left corners shows that $r V=\bar{\lambda} r$, so $r=0$ since otherwise $\lambda \in \sigma_{p}\left(V^{*}\right)$, contrary to the choice of $\lambda$. Similarly $B$ commuting with $W_{\lambda}^{*}$ implies $c=0$, so $B=\operatorname{diag}\{A, \mu\}$ for $B \in C^{*}(W)^{\prime}$. However, since $W_{\lambda}^{n}$ has $V^{n}$ as its upper left block (and similarly for $W_{\lambda}^{* n}$ ), and $V$ is a partial isometry that generates $B(\mathcal{H}), A$ must be a scalar. The identity $B W_{\lambda}=W_{\lambda} B$ also implies $A h=\mu h$, and since $A$ is a scalar and $h$ is nonzero we have $B \in \mathbb{C} I_{B\left(\mathcal{H}_{1}\right)}$. Mapping $v$ to $W_{\lambda}$ defines an irreducible representation $\pi_{\lambda}$ of $\mathcal{P}$ on $\mathcal{H}_{1}$.

If $\pi_{\lambda}$ is unitarily equivalent to $\pi_{\mu}$ for $\lambda, \mu \notin \sigma_{p}\left(V^{*}\right)$, then $W_{\lambda}$ (with spectrum $\sigma(V) \cup\{\bar{\lambda}\})$ and $W_{\mu}$ are unitarily equivalent, so $\lambda=\mu$.

We have not investigated how the choice of $h$ influences the unitary equivalence class of the representation; however, if the dimension of $I_{B(\mathcal{H})}-V V^{*}$ is 1 , then there is up to a scalar in $\mathbb{T}$ only one choice, and these are all in the same representation class under unitary equivalence.

For $\operatorname{dim} \mathcal{H}=2, \alpha \in \mathbb{T}$ and $t \in(0, \pi / 2), v=\left[\begin{array}{lll}\alpha \cos t & 0 \\ \alpha \sin t & 0\end{array}\right]$ is a partial isometry with initial projection $e=\operatorname{diag}\{1,0\}$ and final projection $f \neq e$. Since $e \wedge f=$ $e^{\perp} \wedge f=e \wedge f^{\perp}=0$ while $e \vee f=I_{B(\mathcal{H})}$, the von Neumann algebra (and therefore the $\mathrm{C}^{*}$-algebra) generated by $e$ and $f$ is $B(\mathcal{H})([\mathrm{T}])$. Some computations will reveal that up to unitary equivalence these, along with $\left[\begin{array}{ll}0 & 0 \\ 1 & 0\end{array}\right]$, are all of the distinct irreducible partial isometries on $\mathcal{H}$ with $e$ as initial space. Iterating the above procedure for obtaining irreducible partial isometries on spaces of one dimension greater, we obtain many unitarily inequivalent irreducible representations of $\mathcal{P}$ on any finite dimensional Hilbert space.

Notice that these procedures supply many possibilities for constructing irreducible partial isometries on spaces; for any given such on $\mathcal{H}$, one can find another such on a space of dimension either $2 \operatorname{dim}(\mathcal{H})$ or on a space of $\operatorname{dimension} \operatorname{dim}(\mathcal{H})+1$. The first will have initial (and final) projection with $\operatorname{rank} \operatorname{dim}(\mathcal{H})$, while the second will have initial projection of rank one greater than the rank of the original initial projection. 


\section{3. $K$-GROUPS}

For a $\mathrm{C}^{*}$-algebra $\mathcal{A}$ denote by $V(\mathcal{A})$ the semigroup of equivalence classes of projections in $\bigcup \mathrm{M}_{n}(\mathcal{A})$.

Theorem 3.1. The unital $C^{*}$-algebra $\mathcal{D}$ is homotopy equivalent to $\mathbb{C}$. We have $V(\mathcal{D}) \cong \mathbb{Z}^{+}$and $K_{1}(\mathcal{D})=0$.

Proof. There are unital ${ }^{*}$-homomorphisms $\alpha: \mathbb{C} \rightarrow \mathcal{D}$ and $\beta: \mathcal{D} \rightarrow \mathbb{C}$ with $\alpha$ the canonical unital injection and $\beta$ the unital map, defined via the universal property, mapping the nonunital generator $a$ of $\mathcal{D}$ to 0 . We have $\beta \circ \alpha=I d_{\mathbb{C}}$, while $\alpha \circ \beta$ : $\mathcal{D} \rightarrow \mathcal{D}$ is the unital *-homomorphism sending $a$ to 0 .

Consider the $\mathrm{C}^{*}$-algebra $C^{\sim \mathcal{D}}=\left\{f:[0,1] \rightarrow \mathcal{D} \mid f(0) \in \mathbb{C} 1_{\mathcal{D}}\right\}$, the unitization of the cone $C \mathcal{D}=C_{0}((0,1]) \otimes \mathcal{D}$ over $\mathcal{D}$. With a the nonunital generator of $\mathcal{D}$, the element $b(t)=t a, t \in[0,1]$ of $C^{\sim \mathcal{D}}$ satisfies $\|b\|=\|a\|$, so there is a unital *-homomorphism $\phi: \mathcal{D} \rightarrow C^{\sim} \mathcal{D}$ with $\phi(a)=b$. For $t \in[0,1]$ set $\pi_{t}: C^{\sim} \mathcal{D} \rightarrow \mathcal{D}$ to be the unital *-homomorphisms given by evaluation at $t$. Notice that $\pi_{1} \circ \phi=I d_{\mathcal{D}}$ while $\pi_{0} \circ \phi=\alpha \circ \beta$, so $\mathcal{D}$ is homotopy equivalent to $\mathbb{C}([\mathrm{B}])$.

Thus by Theorem 2.1 we also have $K_{0}(\mathcal{P})=\mathbb{Z}$ and $K_{1}(\mathcal{P})=0$. The above result also implies that $\mathcal{P}$ is stably homotopy equivalent to $\mathcal{K}$; i.e., $\mathcal{P} \otimes \mathcal{K}$ is homotopy equivalent to $\mathcal{K}$. Note however that $\mathcal{P}$ itself is not homotopy equivalent to $\mathcal{K}$, since, as noted after Theorem 2.2 there are no nonzero ${ }^{*}$-homomorphisms mapping $\mathcal{K}$ to $\mathcal{P}$.

We consider some ideals of $\mathcal{P}$ with well-known quotients. Denote the ideal of $\mathcal{P}$ generated by $p-q=v^{*} v-v v^{*}$ by $\mathcal{J}$, and the ideal generated by $(p-q) v=p v-v$ by $\mathcal{I}$. We have $\mathcal{I} \subseteq \mathcal{J}, \mathcal{P} / \mathcal{J} \cong C(\mathbb{T}), \mathcal{P} / \mathcal{I} \cong \mathcal{T}$, the Toeplitz algebra, with the equivalence class of $p$ the unit, while the ideal $\mathcal{J} / \mathcal{I}$ is the ideal in $\mathcal{P} / \mathcal{I}$ generated by (the equivalence class of) $p-v v^{*}$, so is isomorphic to the compacts $\mathcal{K}$. The ideal $\mathcal{P}_{0}$ generated by $v-v^{*} v$ contains $\mathcal{J}$ since $p-q=\left(v^{*}-v\right) v-v\left(v^{*}-v\right)$ and $\left(v^{*}-v\right)=\left(v^{*}-v^{*} v\right)-\left(v-v^{*} v\right)$. The quotient $\mathcal{P} / \mathcal{P}_{0} \cong \mathbb{C}$.

Theorem 3.2. $K_{0}(\mathcal{J})=\mathbb{Z}$ and $K_{1}(\mathcal{J})=0$, while $K_{*}(\mathcal{I})=0$ and $K_{*}\left(\mathcal{P}_{0}\right)=0$.

Proof. If $\mathcal{F}$ is an ideal of $\mathcal{P}$ with $\mathcal{P} / \mathcal{F}$ unital and with quotient map $\pi: \mathcal{P} \rightarrow \mathcal{P} / \mathcal{F}$ mapping $p$ (or $q)$ to the unit, and if $K_{0}(\mathcal{P} / \mathcal{F}) \cong \mathbb{Z}$ is generated by the class of the unit, then the map $K_{0}(\pi): K_{0}(\mathcal{P}) \rightarrow K_{0}(\mathcal{P} / \mathcal{F})$ is surjective, and so an isomorphism. The six-term exact sequence for the ideal $\mathcal{F}$ yields that $K_{1}(\mathcal{F})=0$ and $K_{0}(\mathcal{F}) \cong K_{1}(\mathcal{P} / \mathcal{F})$. Since $K_{1}(C(\mathbb{T})) \cong \mathbb{Z}$ and $K_{1}(\mathcal{T})=K_{1}(\mathbb{C})=0$, the results follow for the various ideals $\mathcal{F}$.

Corollary 3. The group $K_{0}(\mathcal{P}) \cong \mathbb{Z}$ is generated by $[p]$.

Similar arguments show there are ideals of $\mathcal{P}$ with $K_{1}$ group zero and $K_{0}$ group any finite cyclic group.

We examine the projections of $\mathcal{P}$ in more detail. The existence of a faithful trace on $\mathcal{P}$ implies that the $K_{0}$ class of a nonzero projection must be nonzero, so if $r$ is a nonzero projection in $\mathcal{P}$, then $[r]=b[p]$ for some nonzero integer $b$.

Theorem 3.3. If $r$ is a nonzero projection in $\mathcal{P}$, then $[p]=[r]$ in $K_{0}(\mathcal{P})$.

Proof. Since the finite dimensional representations of $\mathcal{P}$ separate points, there is such a representation $\pi$ with $\pi(r)$ a nonzero projection in some $\mathrm{M}_{n}(\mathbb{C})$. We may assume that $\pi$ is nondegenerate. Since $\pi(p)=\pi(\nu)^{*} \pi(\nu)$ we must have that $\pi(p)$ 
is also nonzero. The previous corollary implies that there is a $b \in \mathbb{Z}$ with $[r]=b[p]$ in $K_{0}(\mathcal{P})$. Applying the induced map $\pi_{*}$ on $K_{0}$ we have $[\pi(r)]=b[\pi(p)]$ in $K_{0}(\mathbb{C})$, and $\operatorname{dim} \pi(r)=b \operatorname{dim} \pi(p)$. In particular $b$ must be positive, and so $\rho(r)$ is nonzero for any nondegenerate finite dimensional representation $\rho$. Since $n / 2 \leq \operatorname{dim} \pi(p)$ while $\operatorname{dim} \pi(r) \leq n$, we have that $b=1$ or $b=2$.

If $b=2$ for some projection $r$ in $\mathcal{P}$, then $\rho(r)=I d_{\mathcal{H}_{\rho}}$ for any nondegenerate finite dimensional representation $\rho$, and $\operatorname{dim} \rho(p)=\operatorname{dim}\left(\mathcal{H}_{\rho}\right) / 2$ for any such $\rho$. However, this already fails to be true for an irreducible representation on a Hilbert space of dimension 3. Thus $b=1$ and $[p]=[r]$ (and $\operatorname{dim} \pi(r)=\operatorname{dim} \pi(p)$ ).

Corollary 4. The algebra $\mathcal{P}$ is not unital.

Proof. Choose a nondegenerate finite dimensional representation $\pi$ of $\mathcal{P}$ on $\mathcal{H}$, with $\pi(p) \neq I d_{\mathcal{H}}$, for example an irreducible representation with $\operatorname{dim}(\mathcal{H})>1$. If $\mathcal{P}$ had a unit, then it, and therefore also $p$, would be mapped to $I d_{\mathcal{H}}$.

Corollary 5. Any nonzero projection in $\mathcal{P}$ is Murray-von Neumann equivalent to $p$.

Proof. Since the $\mathrm{C}^{*}$-algebra $\mathcal{D}$ satisfies cancellation of projections (Theorem 3.1 ), so does $\mathcal{P}$ (Theorem 2.1). Now apply Theorem 3.3.

Since $\mathcal{P}$ is residually finite dimensional, so is $\widetilde{\mathcal{P}}$, and thus $\widetilde{\mathcal{P}}$ is stably finite $([\mathrm{B}])$.

There remain several potentially interesting avenues to investigate concerning the $\mathrm{C}^{*}$-algebra $\mathcal{P}$. For example, little is explored concerning the intrinsic dynamics of the algebra $\mathcal{P}$. We note that there is a natural $\mathbb{Z}_{2}$ grading on the algebra $\mathcal{P}$ arising from the order two ${ }^{*}$-automorphism $\alpha$ mapping $v$ to $v^{*}$. There is also a canonical action $\gamma$ by ${ }^{*}$-automorphisms of the group $\mathbb{T}$, with $\gamma_{t}(v)=t v$ for $t \in \mathbb{T}$. Since $\alpha \circ \gamma_{t}=\gamma_{h(t)} \circ \alpha$ with $h$ the homeomorphism $t \rightarrow \bar{t}$ of $\mathbb{T}$, the group $\mathbb{T} \rtimes_{h} \mathbb{Z}_{2}$ acts by automorphisms of $\mathcal{P}$ and acts trivially on $K_{*}(\mathcal{P})$. Moreover these automorphisms leave the ideal $\mathcal{J}$ invariant, so in particular they cannot be inner as they drop to nontrivial automorphisms on the quotient $C(\mathbb{T})$.

\section{ACKNOWLEDGMENTS}

The authors are grateful to the referee for pointing out that a modification of our original proof for Theorem 3.1 would yield a $\mathrm{C}^{*}$-algebra homotopy result.

The authors gratefully acknowledge support, in connection with this research, from the Natural Sciences and Engineering Research Council of Canada, the Pacific Institute of the Mathematical Sciences, and the Fields Institute.

\section{REFERENCES}

[B] B. Blackadar, Theory of $\mathrm{C}^{*}$-algebras and von Neumann Algebras, Encyclopaedia of Mathematical Sciences, 122. Operator Algebras and Non-commutative Geometry 7, SpringerVerlag, Berlin, 2006. MR2188261 (2006k:46082)

[Ch] M.D. Choi, The full $\mathrm{C}^{*}$-algebra of the free group on two generators, Pacific J. Math. 87 (1980), 41-48. MR590864 (82b:46069)

[HM] P. R. Halmos, J. E. McLaughlin, Partial isometries, Pacific J. Math. 13 (1963), 585-596. MR0157241(28:477)

[KR] R. V. Kadison, J. Ringrose, Fundamentals of the Theory of Operator Algebras, Vol. I, Academic Press, New York, 1983. MR719020 (85j:46099)

[OZ] C. Olsen, W. Zame, Some $\mathrm{C}^{*}$-algebras with a single generator, Trans. Amer. Math. Soc. 215 (1976), 205-217. MR.0388114(52:8951) 
[P] C. Pearcy, On certain Von Neumann algebras which are generated by partial isometries, Proc. Amer. Math. Soc. 15, No. 3 (1964), 393-395. MR.0161172 (28:4380)

[R] I. Raeburn, Graph Algebras, CBMS Regional Conference Series in Math., vol. 103, AMS, Providence, Rhode Island, 2005. MR2135030 (2005k:46141)

[T] M. Takesaki, Theory of Operator Algebras, Springer-Verlag, New York, 1979. MR.548728 (81e:46038)

Department of Mathematics and Statistics, University of Calgary, Calgary, aB T2N 1N4, CANADA

Department of Mathematics and Statistics, Memorial University of Newfoundland, St. Johns, NL A1C 5S7, Canada 